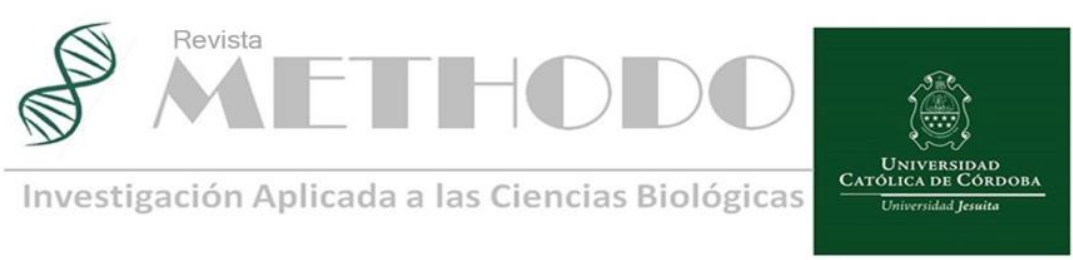

ARTICULO DE REVISION Rev. Methodo 2020;5(2):70-72 https://doi.org/10.22529/me.2020.5(2)06

Recibido 12 Dic. 2019 | Aceptado 21 Feb. 2020 |Publicado 20 Abr. 2020

\title{
Actualización sobre factores de riesgo asociados a daño corneal post Cross-Linking
}

\section{Update on risk factors associated with corneal damage post Cross-Linking}

\author{
Juan C González Castellanos ${ }^{1,2}$, Matías Osaba ${ }^{1,2}$, Virginia Reviglio ${ }^{2}$, Marcela T Canchi ${ }^{1,2}$, Maitén C \\ Arrigone $^{1,2}$, Víctor E Reviglio ${ }^{1,2}$. \\ 1. Instituto de la Visión Cerro de las Rosas, Sanatorio Allende - Sede Cerro, Córdoba, Argentina. \\ 2. Universidad Católica de Córdoba. Facultad de Ciencias de la Salud. Córdoba, Argentina. \\ Correspondencia: González Castellanos Juan Cruz, Instituto de la Visión Cerro de las Rosas, Av. Rafael Núñez 5019 Córdoba, Argentina. E-mail: \\ Juancgonzalezcastellanos@gmail.com.
}

\section{Resumen}

El Cross-Linking corneal es un procedimiento utilizado en oftalmología principalmente para el tratamiento del queratocono, la ectasia corneal más frecuente. A pesar de su baja tasa de complicaciones, no es una técnica exenta de ellas. Entre las principales complicaciones se encuentran el haze y el melting corneal, ambas poseen una fisiopatología que no está totalmente esclarecida.

Se realizó una revisión bibliográfica sobre la asociación entre el uso de quinolonas y el daño corneal frente a la exposición de luz UV. También se plantearon factores inherentes al paciente que se han relacionado al aumento de complicaciones.

Se determinó que la radiación UV produce daños en aquellos tejidos sometidos previamente a tratamiento con quinolonas siendo de suma importancia la correcta anamnesis para seleccionar a los candidatos al procedimiento.

Palabras clave: Cross-Linking corneal, terapia fotodinámica, riboflavina, reacciones adversas, melting corneal.

\begin{abstract}
Corneal Cross-Linking is a procedure used in ophthalmology mainly for the treatment of keratoconus, the most frequent corneal ectasia. Despite its low complication rate, it is not an exempt technique. Among the main complications are haze and corneal melting, both have a pathophysiology that is not fully clarified. A bibliographic review of the association between the use of quinolones and corneal damage versus UV light exposure was performed. Inherent patient factors that have been related to increased complications were also raised.

It was determined that UV radiation causes damage to these tissues, sometimes prior to quinolone treatment, with the correction of the anamnesis being of utmost importance to select the candidates for the procedure.
\end{abstract}

Keywords: corneal Cross-Linking, photodynamic therapy, riboflavin, adverse reactions, corneal melting.

Revista Methodo: Investigación Aplicada a las Ciencias Biológicas. Universidad Católica de Córdoba Jacinto Ríos 571 Bo Gral. Paz. X5004FXS. Córdoba. Argentina. Tel.: (54) 3514517299 / Correo: methodo@ucc.edu.ar / Web: methodo.ucc.edu.ar | ARTICULO DE REVISION Rev. Methodo 2020;5 (2):70-72 


\section{Introducción}

El Cross-Linking corneal (CXL por sus siglas en inglés) es un tratamiento que genera endurecimiento de la córnea. En el campo de la oftalmología se ha utilizado el CXL principalmente como tratamiento para retardar y detener la progresión del queratocono, la ectasia corneal más frecuente. El queratocono genera distorsión de imagen y disminución visual progresiva con adelgazamiento del espesor corneal. Asimismo, el CXL también se emplea en otras enfermedades ectásicas, como tratamiento en queratitis infecciosas y para aclarar cicatrices corneales ${ }^{1}$.

El protocolo estándar del procedimiento se comprende de dos etapas. La primera consiste en instilar una gota de riboflavina (vitamina B2, 402,7 $\mathrm{mOsm} / \mathrm{L}$ ) cada 2 minutos durante 30 minutos. Durante la segunda etapa, continúa la instilación de riboflavina mientras simultáneamente se irradia la superficie ocular con luz ultravioleta A (3 $\mathrm{mW} / \mathrm{cm} 2$, 5.4 Joules/cm2; y 370 nanómetros) durante 30 minutos adicionales para estimular las propiedades fotosensibles de la riboflavina ${ }^{2}$.

Cuando la riboflavina es irradiada con luz ultravioleta A (UVA) se exita y genera un estado de activación que produce radicales libres (especies reactivas de oxígeno o ROS y oxígeno singletes) que catalizan la formación de enlaces covalentes adicionales entre las moléculas del colágeno corneal, fenómeno que genera mayor estabilidad y rigidez en el tejido. Entre los resultados no deseados de este procedimiento se encuentran las infecciones, la hiperplasia epitelial nodular, formación de haze (neblina) en el estroma posterior y melting corneal. En cuanto a la fisiopatología del haze y melting corneal, aún no se han podido esclarecer los procesos que lo generan.

La mayoría de los protocolos para CXL incluyen tratamiento pre y post quirúrgico con antibióticos, preferentemente las fluoroquinolonas de última generación ${ }^{3}$. Esta familia de antibióticos tiene sustituyentes halógenos en la posición del carbono 6, carbono 8 y aminas heterocíclicas en posición 7 . En estas regiones moleculares mencionadas, las fluoroquinolonas tienen capacidad para absorber luz en el rango de los 350 a 435 nanómetros desencadenando un fenómeno de fotodescomposición ${ }^{4}$.

Cuando las fluoroquinolonas absorben la radiación ultravioleta (UV) su fotodescomposición produce tripletes de larga duración que reaccionan con oxígeno y producen radicales libres de oxígeno. La producción fotoquímica del oxígeno atómico o superóxido puede dañar tejidos produciendo necrosis, apoptosis, pérdida de la viabilidad celular y daño de membranas celulares 5

En la literatura se mencionan además otros factores de riesgo que pueden desencadenar complicaciones post CXL. Entre los mencionados, se destacan el síndrome de ojo seco, blefaritis no resuelta, infecciones previas (principalmente HSV), presencia de antígeno HLA-b27, uso de lentes de contacto terapéutico y cirugías previas ${ }^{6}$. Debido a que el CXL es un procedimiento que en los últimos años ha tomado gran importancia en el manejo de la patología oftalmológica, conocer cuáles son las causas de sus complicaciones es importante para poder disminuir la frecuencia de las mismas.

El objetivo de este reporte breve es hacer una revisión bibliográfica detallando los distintos factores relacionados con el incremento de efectos no deseados del CXL.

\section{Discusión}

Las fluoroquinolonas son una familia de agentes antibacterianos de amplio espectro habitualmente utilizadas para el tratamiento de infecciones oculares y de manera profiláctica en procedimiento quirúrgicos para prevenir procesos infecciosos ${ }^{7}$. En 2017 Reviglio y col. demostraron en un modelo animal que la gatifloxacina expuesta a radiación UVA produce efectos lesivos a nivel histológico en el $100 \%$ del grupo estudiado. Esto puede traducirse como opacidad corneal, apoptosis celular, edema tisular y pérdida de transparencia y por consiguiente disminución de agudeza visual ${ }^{8}$. Como alternativa al tratamiento antimicrobiano con quinolonas, se podría usar la Azitromicina ya que no produce alteraciones de la superficie corneal al ser expuesta a la luz UVA y que tiene un amplio espectro para bacterias Gram positivos y un espectro moderado para Gram negativos.

En su trabajo, Chiu y col. reportan un caso de melting corneal agresivo con perforación ocular y posterior trasplante de córnea. Dado que una complicación tan severa no suele ser frecuente, al realizar una nueva anamnesis y un examen exhaustivo del paciente se encontró la presencia de espondilitis anquilosante, retraso en la cicatrización, blefaritis y predisposición a la inflamación asociada a su patología autoinmune ${ }^{9}$ Basados en los factores de riesgo anteriormente mencionados se recomienda realizar una anamnesis y un examen biomicroscópico exhaustivo para determinar la presencia de patologías que pudieran predisponer a reacciones inflamatorias exageradas en la superficie ocular.

Revista Methodo: Investigación Aplicada a las Ciencias Biológicas. Universidad Católica de Córdoba. Jacinto Ríos 571 Bo Gral. Paz. X5004FXS.Córdoba. Argentina.Tel.:(54)351 4517299/ Correo: methodo@ucc.edu.ar / Web: methodo.ucc.edu.ar| ARTICULO DE REVISION Rev. Methodo 2020;5 (2):70-72 
Aunque la fisiopatología del haze y el melting corneal aún no se comprende por completo, es posible que un insuficiente examen médico y una selección inadecuada de fármacos tengan una relación directa con la aparición de complicaciones post CXL.

\section{Bibliografía}

1. Thorsrud, Andreas. Corneal collagen crosslinking in vitro: Inhibited regeneration of human limbal epithelial cells after riboflavinultraviolet-A exposur. Journal of Cataract \& Refractive Surgery. 2012, pp. 1072 - 1076.

2. Messmer, Elisabeth. Update on corneal crosslinking for keratoconu. Oman Journal of Ophthalmology. 2013, pp. 8-11.

3. Dhawan, Shikha. Complications of Corneal Collagen Cross-Linking. Journal of Ophthalmology. 2011, pp. 1-5.

4. Wispelwey, Brian. Clinical Implications of Pharmacokinetics and Pharmacodynamics of Fluoroquinolones, Clinica infectious diseases. 2005, pp. 127 - 135.

5. Leyva, Socorro. Fluoroquinolonas. Mecanismos de acción y resistencia, estructura, síntesis y reacciones fisicoquímicas importantes para propiedades medicinale. Boletín de la Sociedad Química de México. 2008. pp. 1-13.
6. Ashwin, Pammal. Collagen cross-linkage: a comprehensive review and directions for future research. The British journal of ophthalmology. 2010, p. 94.

7. Zhao, Baozhong. Detection and Prevention of Ocular Phototoxicity of Ciprofloxacin and Other Fluoroquinolone Antibiotics. Photochemistry and Photobiology. 2010, pp. 798-805.

8. Reviglio VE, Osaba M, Sambuelli G, Kuo IC. Phototoxic Effect of Topical Fluoroquinolones Administered Before Corneal Crosslinking in a Murine Model. J Ocul Pharmacol Ther. 2017, pp. 33(2):73-78.

9. Chiu HH, Sade S, Chew HF. Corneal melt following collagen crosslinking and topography-guided customized ablation treatment for keratoconus. Can J Ophthalmol. 2017, pp. 52(3): e88-e91.

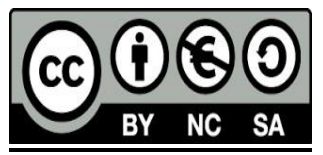

